\title{
All-Optical envelope detection and fiber transmission of wireless signals by external injection of a DFB laser
}

Prince, Kamau; Tafur Monroy, Idelfonso

Published in:

IEEE Photonics Technology Letters

Link to article, DOI:

10.1109/LPT.2008.926915

Publication date:

2008

Document Version

Publisher's PDF, also known as Version of record

Link back to DTU Orbit

Citation (APA):

Prince, K., \& Tafur Monroy, I. (2008). All-Optical envelope detection and fiber transmission of wireless signals by external injection of a DFB laser. IEEE Photonics Technology Letters, 20(15), 1317-1319.

https://doi.org/10.1109/LPT.2008.926915

\section{General rights}

Copyright and moral rights for the publications made accessible in the public portal are retained by the authors and/or other copyright owners and it is a condition of accessing publications that users recognise and abide by the legal requirements associated with these rights.

- Users may download and print one copy of any publication from the public portal for the purpose of private study or research.

- You may not further distribute the material or use it for any profit-making activity or commercial gain

- You may freely distribute the URL identifying the publication in the public portal 


\title{
All-Optical Envelope Detection and Fiber Transmission of Wireless Signals by External Injection of a DFB Laser
}

\author{
Kamau Prince, Student Member, IEEE, and I. Tafur Monroy, Member, IEEE
}

\begin{abstract}
We outline a novel method for all-optical envelope detection of wireless signals by exploiting cross-gain modulation effects in a distributed feedback laser operating with optical injection. We successfully demonstrate envelope detection of a $20-\mathbf{G H z}$ carrier amplitude-shift-keying modulated signal at $2.5 \mathrm{~Gb} / \mathrm{s}$ and its transmission over a 70-km optical fiber link. We present results including bit-error-rate measurements, signal waveforms, and receiver sensitivity penalties associated with envelope detection and fiber transmission.
\end{abstract}

Index Terms-All-optical signal processing, envelope detection, optical access network, radio-frequency-over-fiber (RFoF).

\section{INTRODUCTION}

$\mathbf{O}$ PTICAL communications technology has become established as the preferred option for implementing highspeed data transfer within the core and metropolitan network segments. More recently, the development of fiber-to-the-home networks [1] has allowed the delivery of high-bandwidth services directly to the end-user, encouraging the convergence of wired and wireless signal transport into a common optical fiber backbone infrastructure [2].

The cross-gain-modulation (XGM) effect observed when an optical signal from an external source is introduced into the active region of a semiconductor laser had previously been used to detect the presence of a data packet at an optical receiver port [3]. We have demonstrated the application of the XGM effect in a distributed feedback (DFB) laser to achieve all-optical envelope detection of wireless signals and have reported on experimental characterization of such a device to obtain all-optical envelope detection of an amplitude-shift-keying (ASK) modulated signal with $20-\mathrm{GHz}$ carrier frequency, carrying data at speeds up to $5 \mathrm{~Gb} / \mathrm{s}$ [4]. In this letter, we report on further investigation of the application of the detection scheme in an optical access network environment simultaneously supporting passband radio-frequency-over-fiber (RFoF) and baseband transmission via a $70-\mathrm{km}$ converged wireline and wireless signal transport optical link.

Manuscript received February 8, 2008; revised May 7, 2008. This work was supported by an International Ph.D. Scholarship from the Danish Agency for Science, Technology and Innovation.

The authors are with DTU Fotonik, Technical University of Denmark, Kgs. Lyngby, DK-2800, Denmark (e-mail: kpr@com.dtu.dk).

Color versions of one or more of the figures in this letter are available online at http://ieeexplore.ieee.org.

Digital Object Identifier 10.1109/LPT.2008.926915
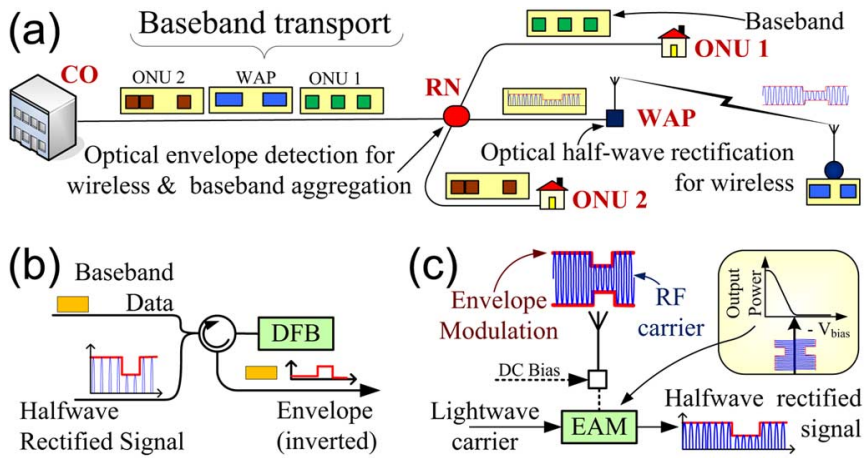

Fig. 1. Optical access network under consideration. (a) Data signals sent between $\mathrm{CO}$ and $\mathrm{ONU}$ and WAP nodes are distributed via intermediate RN. (b) Externally optical injected DFB laser at RN performs all-optical envelope detection of baseband and RoF signals. (c) ASK-modulated RF signal received at WAP, halfwave rectified by EAM and transmitted over optical network.

Section II contains a brief system description and a summary of the conditions under which the experimental study was made. Results are presented with samples of the signal waveforms as well as the receiver sensitivity obtained at various points within the network. The receiver sensitivity analysis, for a target biterror rate (BER) of $10^{-9}$, reveals that less than $1.7-\mathrm{dB}$ receiver sensitivity penalty is associated with fiber transmission for a system operating at $2.5-\mathrm{Gb} / \mathrm{s}$ ASK modulation on a $20-\mathrm{GHz}$ carrier.

\section{SYSTEM DESCRIPTION}

We consider an optical communication network incorporating merged baseband and wireless communications services as shown in Fig. 1(a). Data signals between central office (CO) and optical network unit (ONU) and wireless access point (WAP) nodes are transported via an optical communications network, and distributed at some intermediate remote node (RN). Communication between the ONU and CO terminals is assumed to be formatted for baseband data transmission. WAP nodes interface to wireless access networks and we assume that the signaling between WAP and $\mathrm{CO}$ is formatted for RFoF transmission with the data being modulated onto an appropriate radio-frequency (RF) carrier wave as required for wireless data transmission between WAP and customer node(s). The RN comprises an optically injected DFB laser which transparently processes optical signals from WAP and ONU nodes, and forwards data content to the $\mathrm{CO}$ as shown in Fig. 1(b). This represents a significant simplification in signal processing requirements, and will reduce the overall network 


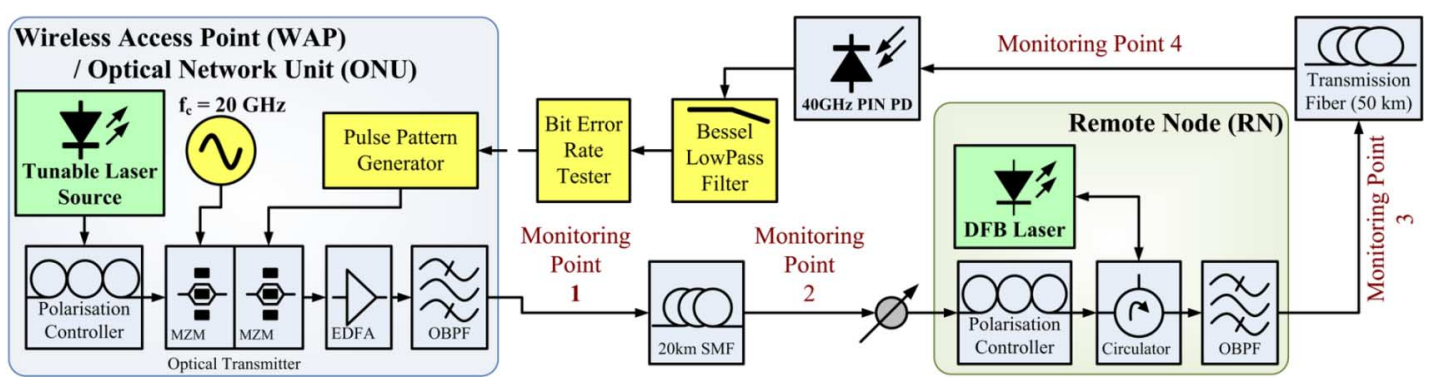

Fig. 2. Experimental setup showing the optical access uplink from WAP/ONU to CO. The RN transparently performs envelope detection and passes baseband traffic to $\mathrm{CO}$ via $50-\mathrm{km}$ transmission fiber. Photodetection and further signal processing occurs at the $\mathrm{CO}$. The transmission fiber was either SMF (dispersion: $17 \mathrm{ps} / \mathrm{nm} / \mathrm{km}$ ) or NZDSF $(5 \mathrm{ps} / \mathrm{nm} / \mathrm{km})$.

costs. Optical half-wave rectification has been achieved by appropriately biasing an electroabsoption modulator (EAM) as shown in Fig. 1(c) [5].

A diagram of the experimental setup is presented as Fig. 2. For experimental purposes, the ASK RFoF/baseband nonreturn-to-zero (NRZ) signaling from the WAP/ONU was provided using a pair of cascaded Mach-Zehnder modulator (MZM) units. Baseband signaling was implemented by disabling the $20-\mathrm{GHz}$ clock signal, this produced an output NRZ pulse stream in response to the digital data input. Activation of the clock input emulated a halfwave-rectified ASK signal at the output: this waveform was typical of that obtained at the output of an EAM-based RF-to-optical half-wave rectifier as shown in Fig. 1(c) and described in [5]. The overall system performance was assessed with the pulse-pattern generator and the BER test set.

Frequency down-conversion of the RFoF signal from passband to baseband is associated with greater robustness of the transmitted signal because of dispersion and power fading effects introduced when an RF signal with a high carrier frequency is sent via an optical network [2]: for example, 6-dB power penalty was observed for $155-\mathrm{Mb} / \mathrm{s}$ data transmission over 50-km single-mode fiber (SMF) when a $29-\mathrm{GHz} \mathrm{RFoF}$ signal was used [6] as opposed to a baseband waveform. We achieved frequency down-conversion using familiar optical components, without specialized RF electrical equipment at WAP or RN. At the RN, XGM effects associated with external optical injection of an active DFB laser were exploited to perform all-optical envelope detection of the ASK-modulated half-wave rectified input. The DFB used in this experiment was an uncooled multiple quantum-well coaxial fiber device: it operates without wavelength control. Since the optical gain in quantum-well devices is polarization-dependent, polarization controllers were used to optimize the XGM effect exploited for all-optical envelope detection. A laser device with bulk material would not require such controllers, as its optical gain is generally less polarization-dependent. A variable optical attenuator was used to maintain uniform injected power to the DFB.

A 20-km span of standard SMF was used between WAP/ONU and RN: $50 \mathrm{~km}$ of transmission fiber was used between $\mathrm{CO}$ and $\mathrm{RN}$; this was alternately implemented with SMF (17-ps $/ \mathrm{nm} / \mathrm{km}$ dispersion at $1550 \mathrm{~nm}$ ) or nonzero dispersion-shifted fiber (NZDSF), with 4-ps/nm/km dispersion. We assessed system performance with pseudorandom binary sequence (PRBS) data of lengths $2^{7}-1$ and $2^{15}-1$ at a bit rate of $2.5 \mathrm{~Gb} / \mathrm{s}$ for baseband and also RFoF signaling. The baseband traffic simulated data flows between CO and ONU, encoded in NRZ pulses. The RFoF signaling would be used for traffic flows to a wireless access network interfaced at the WAP, and was implemented with an optically halfwave rectified ASK modulated $20-\mathrm{GHz}$ carrier wave [5]. Signal waveforms obtained at various points in the network were observed for these types of input. System performance was also assessed using a receiver sensitivity analysis at various monitoring points. For a target BER of $10^{-9}$, the receive sensitivity power penalty incurred by end-to-end transmission via this system was less than 0.7 and $1.6 \mathrm{~dB}$ when NZDSF and SMF, respectively, was used for the 50-km access link.

\section{RESULTS}

\section{A. Signal Propagation Through Network}

With this system, XGM was previously [4] maximized when the wavelength of the injected optical signal into the DFB coincided with one of the spectral side lobes of the DFB output spectrum and was higher than the lase wavelength, and with an average injected power level in the interval $[-4.2,-8.0] \mathrm{dBm}$. In this experiment, the DFB wavelength was approximately $1551 \mathrm{~nm}$; the following results were obtained with a WAP/ONU laser wavelength of $1553 \mathrm{~nm}$ and an average injected power level of $-7 \mathrm{dBm}$ into the DFB at RN.

Sample optical waveforms at WAP and RN are presented as Fig. 3; Fig. 3(a) shows the waveform obtained at WAP output (MP1) and Fig. 3(b) is the corresponding waveform at RN input (MP2). Fig. 3(c) presents a 64-sample average of the waveform obtained at RN output (MP3): the eye diagram at MP3 is presented as Fig. 3(d). We clearly identify the optical halfwave rectified ASK signal from the WAP [in Fig. 3(a)]; note that it is attenuated at MP2 [Fig. 3(b)] and that the lowest part of the waveform during transmission of a "zero" bit does not return to the ground level indicated. In Fig. 3(c), a clear distinction can be made between RN output levels, indicating that the RN has performed envelope detection as desired. This waveform shows an underdamped response, having overshoot and transient oscillation at each rising edge, as consistent with relaxation oscillations typically observed at the output of a directly modulated DFB laser [7]. Some pattern dependence was observed in RN output, 

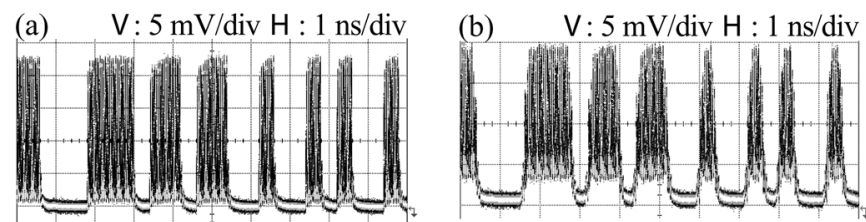

(c)V: $442 \mathrm{~mW} / \mathrm{div} \mathrm{H}: 100 \mathrm{ps} / \mathrm{div}$

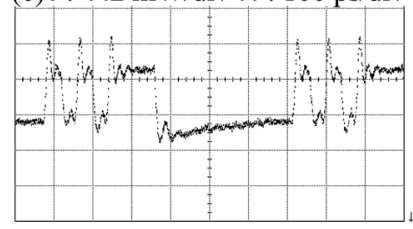

(d) $\mathrm{V}: 5 \mathrm{mV} / \mathrm{div} \mathrm{H}: 100 \mathrm{ps} / \mathrm{div}$

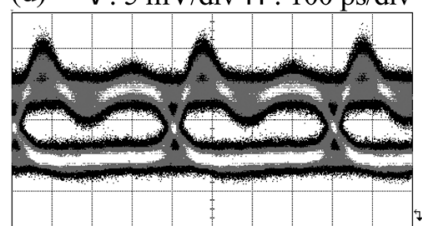

Fig. 3. (a) ASK modulated optical signal present at WAP output, Measuring Point 1 (MP1); (b) at input to RN after 20-km SMF (MP 2): (c) 64-average sample of waveform and (d) eye diagram at RN output (MP3). (a) $\quad \mathrm{V}: 0.5 \mathrm{~mW} / \operatorname{div} \mathrm{H}: 1 \mathrm{~ns} / \mathrm{div}$

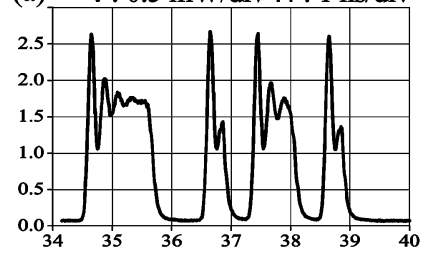

(c) $\mathrm{V}: 50 \mathrm{mV} / \mathrm{div} \mathrm{H}: 100 \mathrm{ps} / \mathrm{div}$

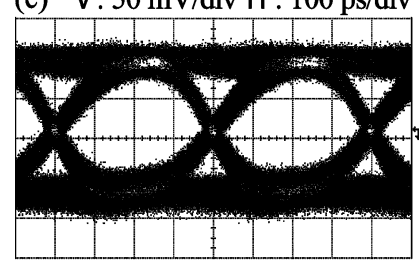

(b) $\quad \mathrm{V}: 0.5 \mathrm{~mW} / \mathrm{div} \mathrm{H}: 1 \mathrm{~ns} / \mathrm{div}$

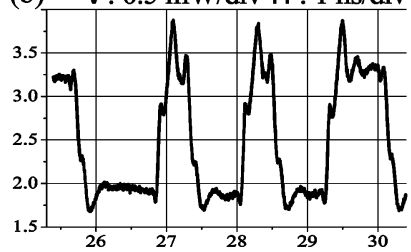

(d) $\mathrm{V}: 5 \mathrm{mV} / \mathrm{div} \mathrm{H}: 100 \mathrm{ps} / \mathrm{div}$

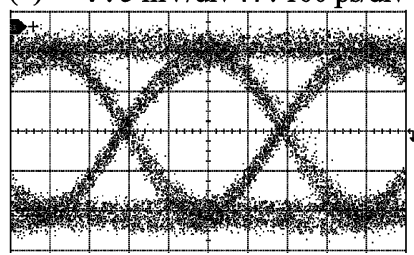

Fig. 4. Signal waveforms obtained at output of long-reach access link (MP4). [(a) and (b)] Sample detected signal observations and [(c) and (d)] post-LPF electric signal eye diagrams are presented when NZDSF and SMF, respectively, were implemented as the 50-km transmission fiber.

with minor level shift in response to a long run of "ones". The eye diagram obtained at MP3 [Fig. 3(d)] shows well-defined level transitions and a good opening. When the 50-km transmission fiber was implemented with NZDSF, the waveform presented in Fig. 4(a) was observed at MP4. We can see that the overshoot and high-frequency transient ripple were removed by electrical postphotodetection filtering using a Bessel low-pass filter (LPF) and the result is presented as Fig. 4(c). The corresponding waveforms obtained when SMF was used are presented as Fig. 4(b) and (d).

\section{B. Receiver Sensitivity Assessment}

We assessed the receiver sensitivity power penalty for a target BER of $10^{-9}$ at various points throughout the system under test: the results are presented in Fig. 5. At baseband, for a desired BER of $10^{-9}$, a receive sensitivity power penalty of less than $0.1 \mathrm{~dB}$ was incurred through the use of the DFB to perform all-optical envelope detection in a back-to-back configuration. The penalty incurred at the end of the $20-\mathrm{km}$ link between WAP/ONU and RN was less than $0.2 \mathrm{~dB}$. Total power penalty incurred by end-to-end transmission over the network link under consideration was less than $0.7 \mathrm{~dB}$ for NZDSF transmission fiber and less than $1.6 \mathrm{~dB}$ for SMF.

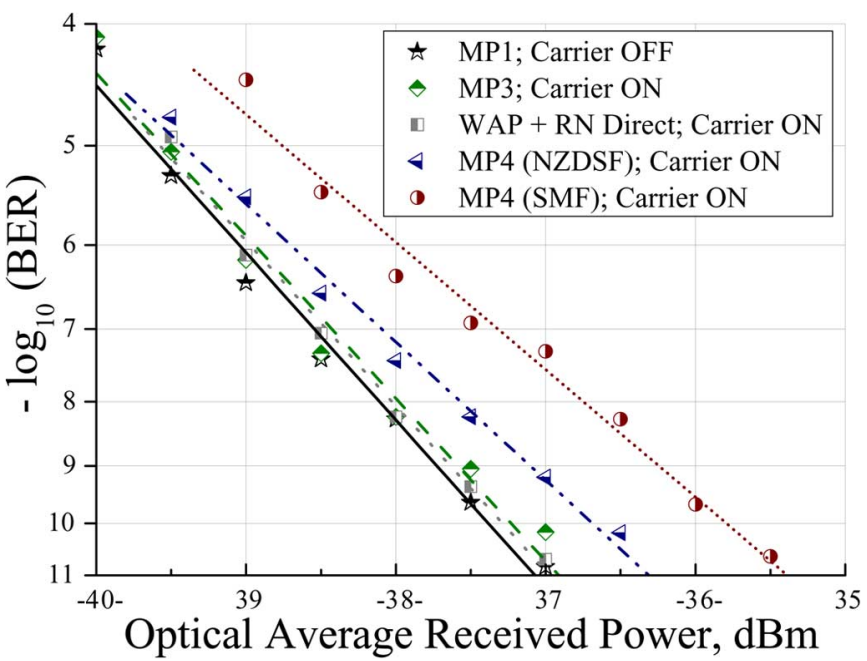

Fig. 5. Receiver sensitivity analysis of the access link system at various points, with a PRBS of length $2^{15}-1$. The baseline is at measuring point (MP) 1 , with the 20-GHz carrier OFF. Carrier-ON receiver sensitivity at MP2 is also presented, and also for direct connection of WAP to RN (WAP + RN) without the 20-km SMF. Results obtained at the access link output are shown for 50-km transmission fiber of SMF and NZDSF type.

\section{CONCLUSION}

We have successfully achieved signal transmission in a converged long-reach access link environment simultaneously supporting both baseband and RoF traffic streams at $2.5 \mathrm{~Gb} / \mathrm{s}$, using only common optical components and simple WAP, ONU, and $\mathrm{RN}$ node design. Successful all-optical envelope detection of an optical halfwave rectified ASK signal with 20-GHz carrier has simultaneously been demonstrated; RF signal frequency downconversion was thus achieved in the optical domain, without specialized electrical high-frequency RF components. Signal quality analysis at various network points has been presented in terms of measured BER, indicating that low receiver sensitivity penalties are associated with envelope detection in a DFB and fiber transmission. These results indicate the potential of the proposed scheme for converged wireless and baseband signal transport in a typical $2.5-\mathrm{Gb} / \mathrm{s}$ optical access network scenario.

\section{REFERENCES}

[1] T. Koonen, "Fiber to the home/fiber to the premises: What, where, and when?," Proc. IEEE, vol. 94, no. 5, pp. 911-934, May 2006.

[2] A. Ng'oma, "Radio-over-fibre technology for broadband wireless communication systems" Ph.D. dissertation, Tech. Univ. Eindhoven, Eindhoven, The Netherlands, Jun. 2005 [Online]. Available: http://alexandria.tue.nl/extra2/200512106.pdf

[3] B. Koch, Z. Hu, J. Bowers, and D. Blumenthal, "All-optical payload envelope detection for variable length $40-\mathrm{Gb} / \mathrm{s}$ optically labeled packets," IEEE Photon. Technol. Lett., vol. 18, no. 17, pp. 1846-1848, Sep. 1, 2006.

[4] K. Prince, I. T. Monroy, J. Seoane, and P. Jeppesen, "All-optical envelope detection for radio-over-fiber links using external optical injection of a DFB laser," Opt. Express, vol. 16, no. 3, pp. 2005-2014, 2008.

[5] I. T. Monroy, J. Seoane, and P. Jeppesen, "All-optical envelope detection for wireless photonic communication," in Proc. ECOC, Berlin, Germany, Sep. 2007.

[6] D. Novak, "Fiber optics in wireless applications," in Proc. Opt. Fiber Commun. Conf. (OFC), Los Angeles, CA, Feb. 22-27, 2004, short course 217.

[7] G. P. Agrawal, Fiber-Optic Communication Systems. New York: Wiley, 2002, sec. 3.5.3. 